\title{
EFFECT OF MELTING ON TARGET PERFORMANCE
}

\author{
F. M. Bieniosek
}

September 10, 1991

\section{Introduction}

The brightness of the antiproton source increases as the proton beam spot size on the target is reduced. The RMS beam spot size may be reduced to below $\sigma_{b}=0.1 \mathrm{~mm}$, before competing sources of emittance limit the achievable yield. At the same time, the density of energy deposition increases rapidly as the radius is reduced. Thus operation of the target at the highest yields subjects the target to very high peak energy deposition $E_{m}$. Fits to the MonteCarlo calculations of target yield and energy deposition from Ref. 1 are plotted in Figure 1. Experience has shown little or no sign of damage in copper targets up to about $500 \mathrm{~J} / \mathrm{g}$. If, as the energy density is increased, rupture of the copper target due to overpressure or shock-induced tensile stress does not occur, the ultimate brightness of the target will be limited by melting of the target material and consequent density depletion. This outcome was anticipated early in development of the antiproton source [Ref. 2]. The current memo addresses the melting problem with the goal of predicting the practical limitations of the target as the proton intensity is increased to $5 \times 10^{12}$ protons per pulse. The predictions are made in a way that can be experimentally tested. They may also help determine the utility of a beam sweeping system.

\section{Melting Point of Copper}

The melting point of copper is a function of pressure. Experimentally, the relationship between pressure and melting temperature is a straight line up to at least $7 \mathrm{GPa}$ [Ref. 3]:

$$
T_{\text {melt }}=T_{m 0}+95 P
$$

where $T_{m 0}=1356 \mathrm{~K}$ is the melting point at atmospheric pressure, and $P(m 0 \mathrm{GPa})$ is the local pressure. The pressure is in turn given by the Gruneisen equation, 


$$
P=\rho \Gamma E
$$

where $\rho=8.96 \mathrm{~g} / \mathrm{cc}$ is the density of copper, $\Gamma=1.96$ is the Gruneisen parameter, and $E$ is the deposited energy $(\mathrm{J} / \mathrm{g})$.

The specific heat of copper is generally quoted for constant pressure:

$$
C_{p}=0.858+1.04 \times 10^{-4} \mathrm{~T} \quad(\mathrm{~J} / \mathrm{g} \mathrm{K})
$$

This may be corrected to give the specific heat at constant volume [Ref. 4]

$$
C_{p}-C_{v}=-T\left(\frac{\partial v}{\partial T}\right)_{P}^{2}\left(\frac{\partial P}{\partial v}\right)_{T}=\frac{\alpha^{2} v T}{\beta_{T}}=6 \times 10^{-5} T
$$

where $\alpha_{p}$ is the volume expansivity, $v$ is the specific volume, and $\beta_{T}$ is the isothermal compressibility (reciprocal of the bulk modulus). Hence, we have

$$
C_{v}=0.858+4.4 \times 10^{-5} \mathrm{~T}
$$

Finally, the energy deposition is related to the temperature by

$$
\begin{aligned}
E= & \int_{s 00}^{T} C_{v} d T \\
& =0.858 T+2.2 \times 10^{-5} T^{2}-109.4
\end{aligned}
$$

assuming initial temperature is $300 \mathrm{~K}=27 \mathrm{C}$.

Equations 1-3 allow calculation of the temperature and state as a function of energy deposition; the results are plotted in Figure 2. At $1702 \mathrm{~K}\left(E_{m}=563 \mathrm{~J} / \mathrm{g}\right)$, melting begins; at $1827 \mathrm{~K}\left(E_{m}=770\right.$ $\mathrm{J} / \mathrm{g}$ ), the local material is completely molten (the latent heat of fusion $H_{f}=207 \mathrm{~J} / \mathrm{g}$ ). The phase transition is rapid. High-speed exploding foil experiments, for example, have shown good agreeement between measured and predicted resistivity of foil material as it passes through solid-liquid-gaseous states on a time scale much shorter than $1 \mu \mathrm{s}$. See Figure 3 for a typical result [Ref, 5],

At $770 \mathrm{~J} / \mathrm{g}$, the pressure is approaching $13.5 \mathrm{GPa}$. The pressure and temperature are sufficiently high to produce synthetic diamonds. 


\section{Density Depletion Model}

As the energy deposition increases beyond the melting point, a liquid channel forms. Mass flows radially out of the (hotter) core to equalize pressure throughout the channel. As the target density drops, the yield drops with it. Hence there is a penalty in yield to proceeding beyond about $800 \mathrm{~J} / \mathrm{g}$ energy deposition on target. This simple model of a partially melted target is illustrated in Figure 4. Here the radius $r$ marks the solid-liquid interface. The model assumes cylindrical symmetry, and neglects axial variations and boundary conditions at the wall. The model is made simple by an assumption of pressure equilibrium, which is justified by the relevant time scales. These are the length of beam spill $\tau_{\text {beam }}=1.6 \mu \mathrm{s}$; the time required for a sound pulse to travel across the channel $\tau_{\text {sound }}=0.15 \mathrm{~mm} \div 4.5 \mathrm{~mm} / \mu \mathrm{s}=33 \mathrm{~ns} ;$ and the time required for conduction of heat away from the channel $\tau_{\text {cond }}=100 \mu \mathrm{s}$ [Ref. 6].

(a) $\tau_{\text {beam }} \gg \tau_{\text {sound }}$ This implies that transient effects are small. Material remains near pressure equilibrium during and after the pulse; there is no non-steady fluid motion after the end of the beam pulse. Energy carried out by the shock wave is neglected.

(b) $\tau_{\text {cond }} \gg>\tau_{\text {beam }}$. Heat conduction is negligible on time scales of interest and is therefore ignored. The channel cools long after the beam has passed.

The density variation of liquid copper with temperature at constant pressure is $d \rho / d T=-1 x 10^{-4} \rho$, and the specific heat of liquid copper is $C_{p}=0.495 \mathrm{~J} / \mathrm{g} \mathrm{K}$ [Ref. 7]. Hence, the density can be written in terms of energy deposition and integrated to give the density profile of liquid copper in the channel

$$
\rho(r)=\rho_{t} \exp \left[-2.02 x 10^{-4}\left(E(r)-E_{t}\right)\right]
$$

where $\rho_{t}$ is the density and $E_{t}$ is the energy deposition at the solidliquid transition. $E_{t}$ is presumably in the range $563-770 \mathrm{~J} / \mathrm{g}$ characterized by partial melting. The uncertainty in $E_{t}$ leads to a corresponding uncertainty in central depletion with respect to the edge.

\section{Model Predictions}

The prediction of density depletion as energy deposition is increased beyond about $800 \mathrm{~J} / \mathrm{g}$ can be tested experimentally. Assuming the normalized instanteous yield to be proportional to the product of target mass density and beam density, integrated over the target, we have

$$
\text { Yield }=\sigma_{b}{ }^{-2} \int_{0}^{\infty} \rho(r) \exp \left(-r^{2} / 2 \sigma_{b}^{2}\right) r d r
$$


where $\rho(r)=\rho_{t}=1$ for $r 2 r_{1}$ and $\rho(r)$ is given by Equation 4 for $r<r_{1^{*}}$ The energy deposition profile is assumed to be Gaussian

$$
E(r)=E_{m} \exp \left(-r^{2} / \sigma_{h}^{2}\right)
$$

where $\sigma_{h} / \sigma_{b} \geq 1$ and $r_{1}=\sigma_{h}\left\{2 \ln \left(E_{m} / E_{t}\right)\right\}^{1 / 2}$. The total yield, integrated over the length of the beam pulse, is then the time integral of the instantaneous yield. This is a consequence of the assumption of pressure equilibrium. A typical result of this calculation, for $E_{t}=770 \mathrm{~J} / \mathrm{g}$, and $\sigma_{h}=1.5 \sigma_{b}$ is plotted in Figure 5. The plot shows that, as deposited energy increases, central density drops rapidly in the liquid state, but the integrated yield drops much more slowly. The over-all target yield is plotted in Figure 6, as a function of beam spot size. Two proton beam intensities are shown, and the relative collection efficiency from Figure 1 is also shown for reference. The variation in the intergrated yield for the range of possible choices for $E$ is also shown. There is a trade-off between the target production and collection efficiency. The optimum point for $5 \times 10^{12}$ protons per pulse is about $93 \%$ of the theoretical yield, which can be obtained at about $\sigma_{b}=0.14 \mathrm{~mm}(1500 \mathrm{~J} / \mathrm{g})$. Of course, it remains to be seen whether the copper target can sustain this energy density for many pulses.

\section{Comments}

Several additional characteristics of the solid-liquid transition can affect the density depletion, but were neglected.

(a) As the copper melts, it expands. The amount of intrinsic expansion can be determined from the slope of the phase transition curve (Equation 1) by means of the Clausius-Clapeyron equation [Ref. 8]. The volume change is about $3-4 \%$ in our case.

(b) Expansion of the liquid channel compresses the solid material surrounding the channel. The pressure naturally acts to compress the channel back toward solid density, roughly cancelling the effect of (a) on the yield.

(c) The solid-liquid transition occurs over a broad range of 207 $\mathrm{J} / \mathrm{g}$ where solid and liquid states coexist. The model neglects the mushy region and assumes a well-defined transition.

\section{References}

1. I. L. Azhgirey, N. V. Mokhov, S. I. Striganov, Antiproton Production for Tevatron, FNAL-TM-1730 (1991).

2. D. B. Cline, The Development of Bright Antiproton Sources and High Density Targeting, 11th International Conference on High Energy Accelerators (July 7-11, 1980). 
3. K. S. Trainor, J. Appl. Phys. 54, 2372 (1983).

4. G. Van-Wylen, R. Sonntag, Fundamentals of Classical Thermodynamics, 1973 , p. 390

5. R. E. Reinovsky, et. al., IEEE Trans. Plasma Science, 10, 73 (1982).

6. V. Visnjic, PBAR Note \#492, Dec. 7, 1990.

7. E. A. Brandes, Smithells Metals Reference Book, 1983, p. 14-7

8. Ref. 4, p. 376 . 



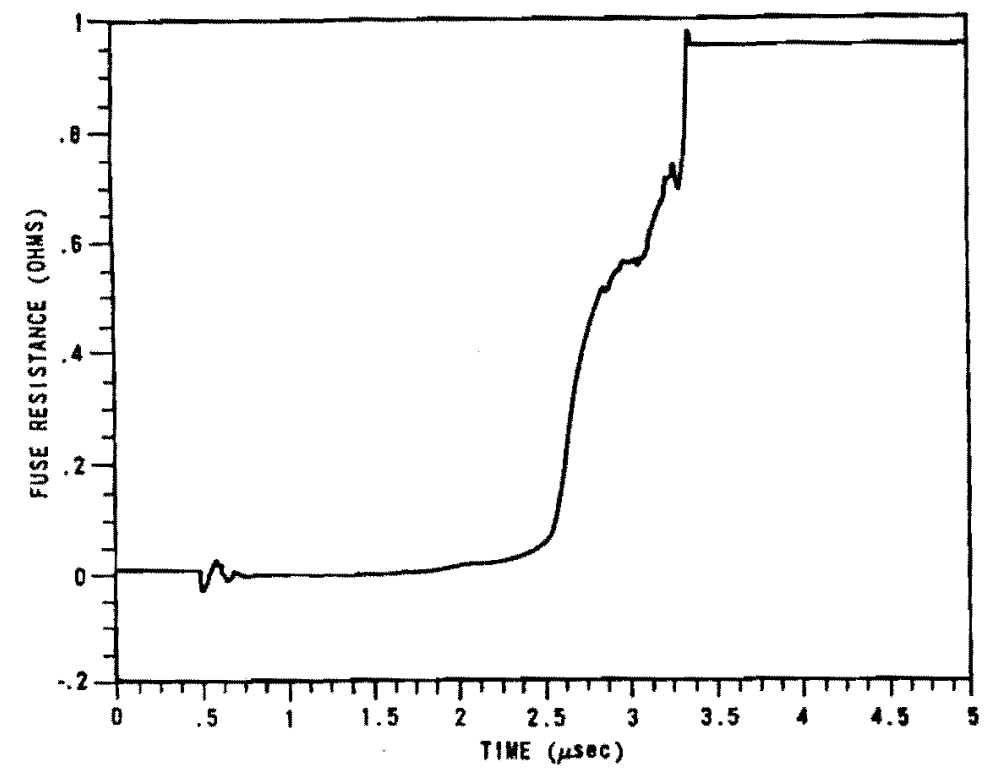

Fig. 9. Resistant increase as a function of time for an aluminum foil use.

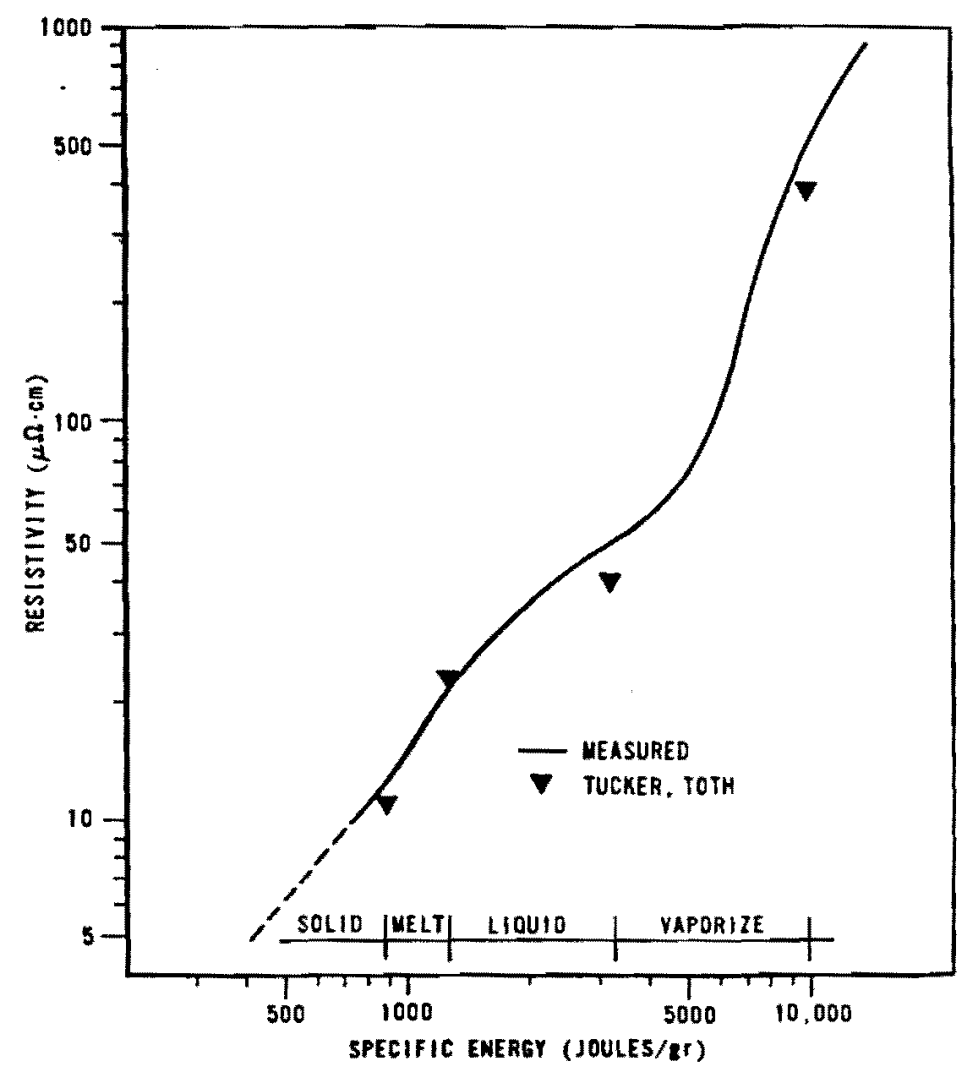

Fig. 10. Resistance as a function of specific energy.

Figure 3. Results of an exploding-foil experiment [Ref. 5]. 


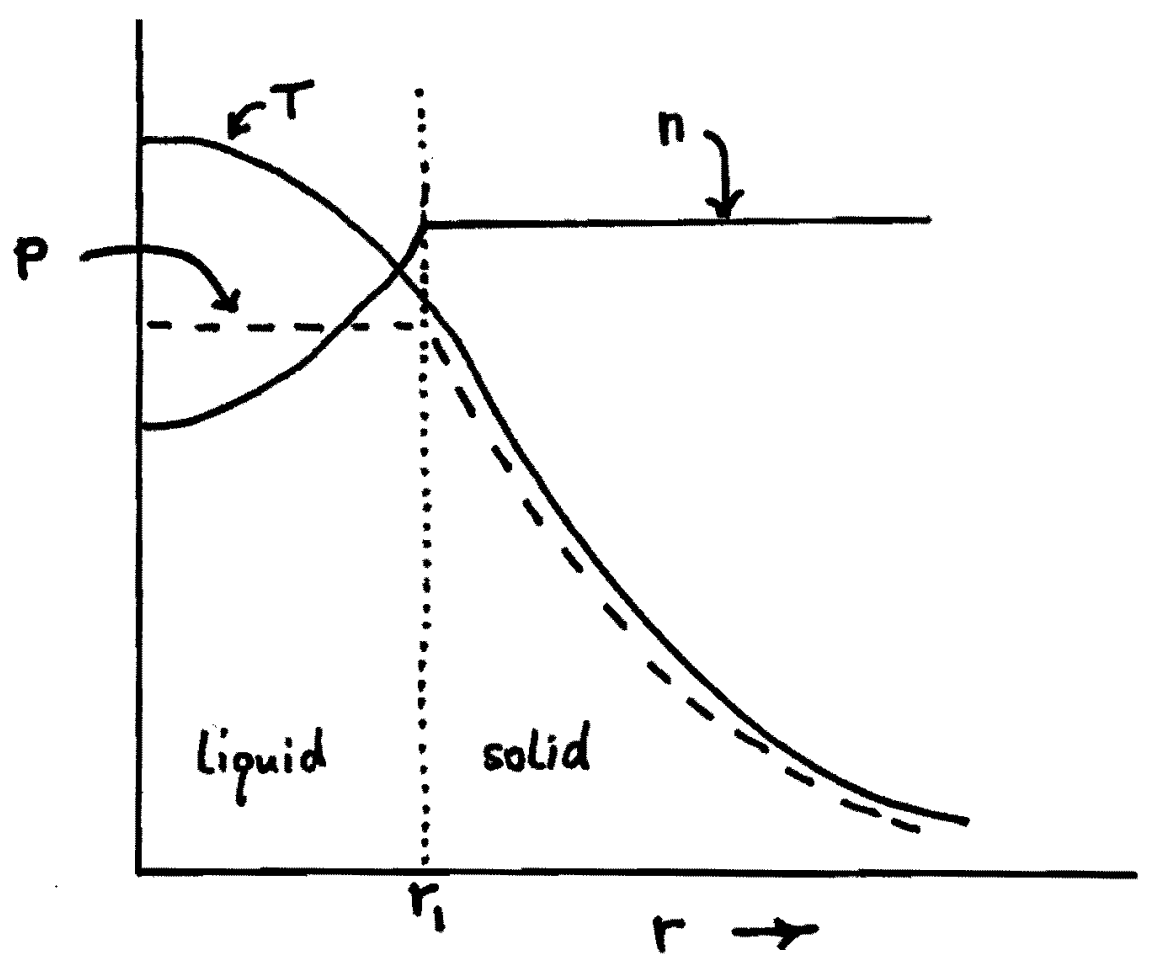

Figure 4. Temperature, density, and pressure profiles in a partiallymelted target. 


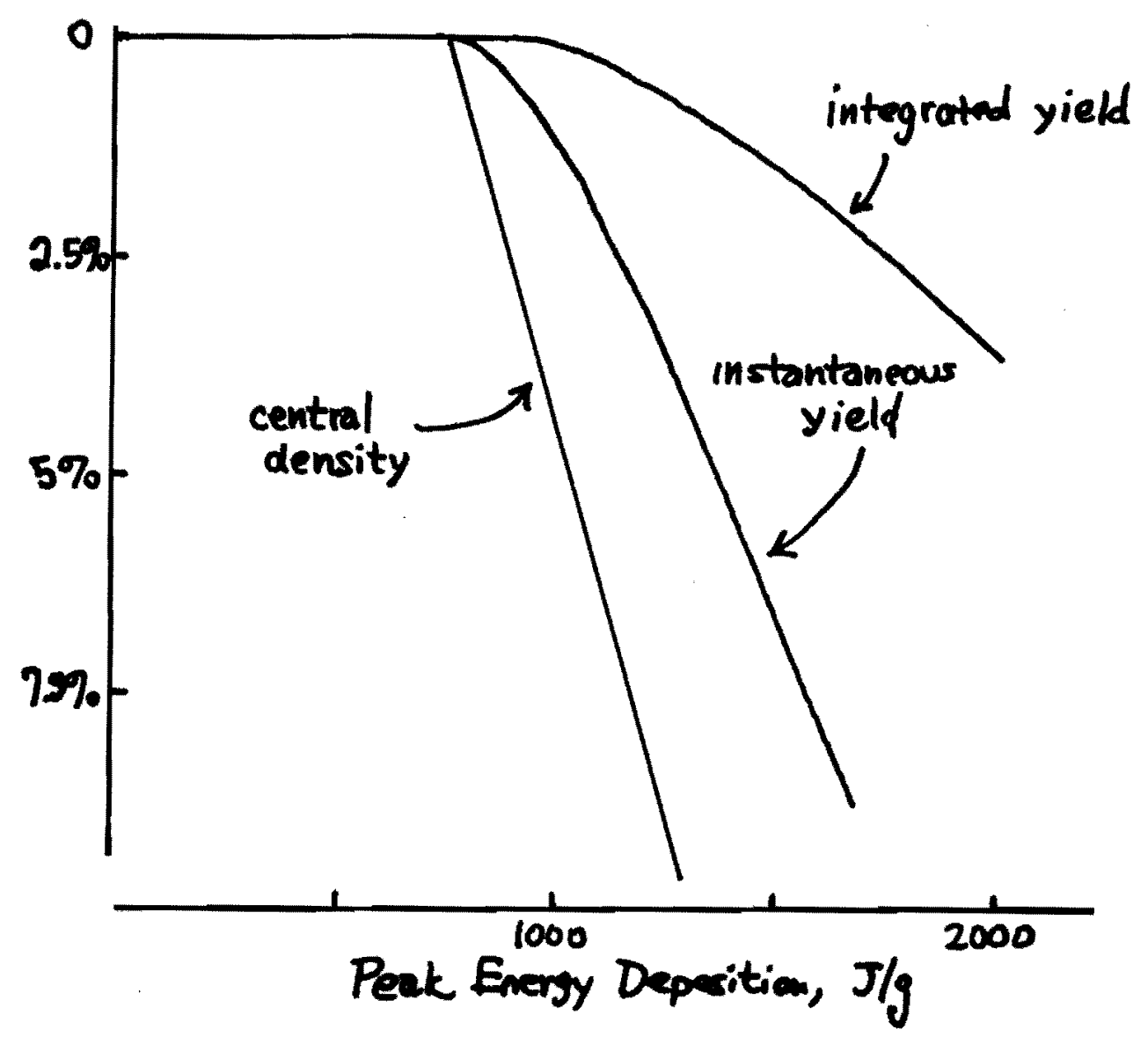

Figure 5. Effect of deposited energy on target density and yield. Calculation assumes $E_{t}=770 \mathrm{~J} / \mathrm{g}$ and $\sigma_{h}=1.5 \sigma_{b}$. 
